\title{
Factors Associated with Osteoporosis Management Among Veterans Who Received Androgen Deprivation Therapy for Prostate Cancer
}

J Gen Intern Med 36(10):3270-2

DOI: $10.1007 / \mathrm{s} 11606-020-06418-5$

(C) This is a U.S. government work and not under copyright protection in the U.S.; foreign copyright protection may apply 2021

\section{INTRODUCTION}

The Veterans Health Administration cares for more than 200,000 men living with prostate cancer. ${ }^{1}$ Many Veterans receive androgen deprivation therapy (ADT) for their $\mathrm{PCa}$ and while ADT can improve PCa clinical outcomes, it can also reduce bone density. Clinical guidelines recommend evaluating men on ADT with densitometry (DXA) in conjunction with pharmacotherapy as part of fracture risk management. ${ }^{2}$ Left untreated, osteoporosis contributes to significant disability and death from fractures. Primary care clinicians, with their comprehensive understanding of patient comorbidities and associated pharmacotherapy, are optimally positioned to refer men at risk from ADT for DXA and to initiate osteoporosis treatment, including bisphosphonates, which can decrease their fracture risk. ${ }^{3}$ Given the increasing population of older Veterans who may use ADT, the objectives of this study were to (1) assess the proportion of men on ADT receiving osteoporosis care; and (2) identify factors associated with receipt of osteoporosis care, including facility characteristics.

\section{METHODS}

Using VHA administrative data, we identified a cohort of men aged 50-99 $(N=31,047)$ with ADT-induced osteoporosis risk (defined as evidence of orchiectomy $(N=1875)$ or at least 3 months of ADT $(N=29,173))$ in the period 2009-2014. We

Received July 9, 2020

Accepted December 8, 2020

Published online January22, 2021 excluded men with evidence of orchiectomy or ADT prior to 2009 ( $n=90)$; end-of-life care $(n=155)$; other bone conditions $(n=179)$; spinal cord injury $(n=194)$; reliance on private sector primary care $(n=400)$; or metastatic cancer $(n$ $=2075)$ in the prior 2 years. Another 70 men were excluded due to missing facility assignment data. Specific data sources and diagnostic codes are available upon request.

Study outcomes were receipt of densitometry (i.e., DXA) and/or osteoporosis medication (e.g., bisphosphonates) within 12 months following ADT exposure. Covariates included patient sociodemographics and comorbidity and facility characteristics reflecting comprehensive cancer care delivery (Tables 1 and 2), and were compared using the chi-square or Fisher exact test as appropriate. Patient-level data were identified using claims incurred during the 12 months prior to the date of initial ADT exposure; characteristics of cancer care at each facility were identified from a national survey. All statistical tests were two-sided and a $p$ value $<0.05$ was used to detect statistical significance. We developed bivariable and multivariable Cox proportional hazards regression models to determine hazards of the outcomes. A hospital random effect was included to account for clustering. Death within the observation period was considered a competing event and censored. For the final multivariable Cox regression model, all variables of interest were included regardless of statistical significance. All analyses were completed in SAS Enterprise Guide 7.1 This research was approved by the institutional review board at the Iowa City VA Health Care System in conjunction with the University of Iowa.

\section{RESULTS}

In this cohort $(N=23,046)$, a minority of men received DXA (6.6\%), osteoporosis medication $(3.5 \%)$, or either 
Table 1 Cohort Characteristics at Baseline

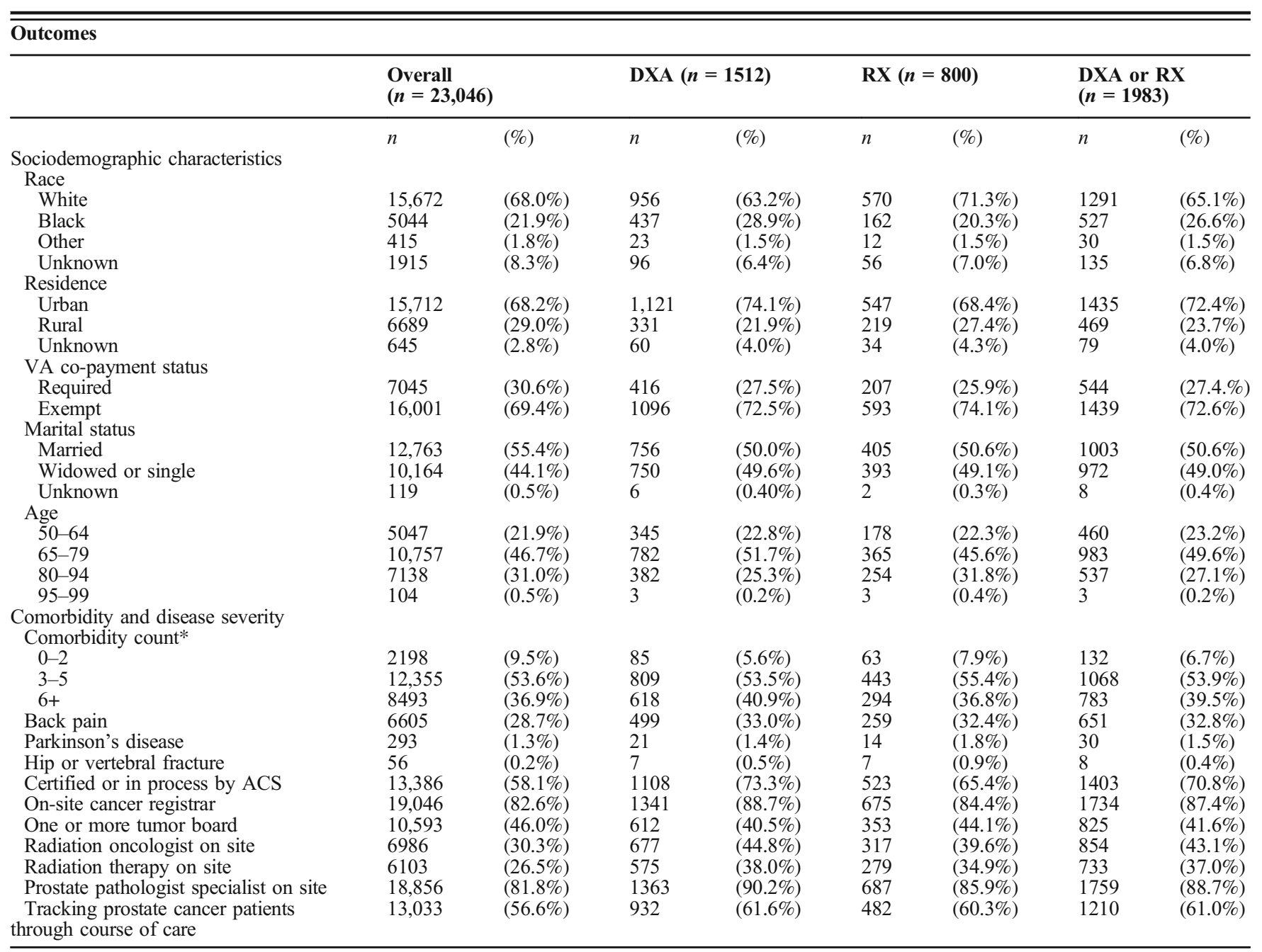

*Sum of comorbidities identified using the Elixhauser approach $^{4}$

marker of care $(8.6 \%)$ (see Table 1$)$. In our final multivariable hazard model (adjusted for year of cohort entry), few factors were shown to affect the likelihood of receiving bone health care after ADT. Men with prior fragility fracture or back pain, who received radiation therapy in addition to ADT, who were single or widowed, who had three or more comorbidities, or who were treated at facilities with an on-site radiation oncologist had higher likelihood of any osteoporosis care. Men residing in rural areas had a lower likelihood of DXA and men required to co-pay for VA services or who were of Black race had a lower likelihood of initiating medications.

\section{DISCUSSION}

Few of these Veterans with fracture risk from ADT received osteoporosis care. Our data do not include cancer stage or DXA results; thus, we may overestimate the number of men with indication for DXA or medication. Our analysis shows that some clinicians are recognizing fracture, disease severity, and back pain as osteoporosis risk and appropriately managing osteoporosis. We identified a positive association between cancer care quality and receipt of bone health care, implying that primary care clinicians should coordinate efforts with oncologists or urologists in managing the potential consequences of ADT in those settings. Bone health care is an important facet of PCa survivorship and further research into patient and provider barriers to appropriate bone health care is warranted. ${ }^{4}$

Acknowledgments: The datasets generated and analyzed for the current study are not publicly available due to VA Privacy and Information Security constraints but may be available from the 
Table 2 Hazard of Receiving Bone Health Care Within 12 Months of Exposure to ADT 2009-2014

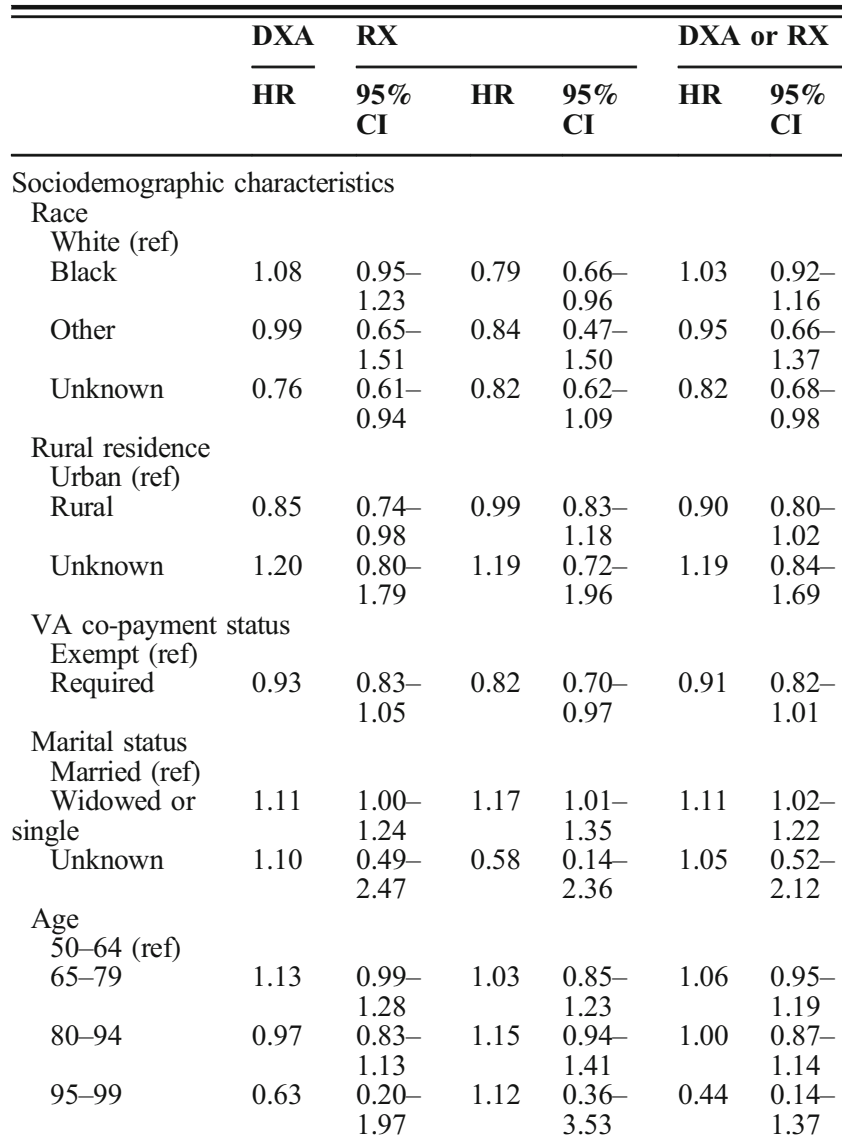

Comorbidity and disease severity

Comorbidity count $*^{5}$

$\begin{array}{cclllll}\begin{array}{c}\text { 0-2 (ref) } \\ 3-5\end{array} & 1.55 & 1.24- & 1.20 & 0.92- & 1.35 & 1.12- \\ & & 1.94 & & 1.57 & & 1.62 \\ 6+ & 1.63 & 1.29- & 1.13 & 0.86- & 1.37 & 1.14- \\ & & 2.05 & & 1.49 & & 1.66 \\ \text { Parkinson's } & 1.13 & 0.74- & 1.44 & 0.84- & 1.26 & 0.88- \\ \text { disease } & & 1.75 & & 2.45 & & 1.81 \\ \text { Back pain } & 1.16 & 1.04- & 1.18 & 1.01- & 1.17 & 1.06- \\ & & 1.30 & & 1.37 & & 1.29 \\ \text { Radiation } & 1.28 & 1.04- & 2.11 & 1.62- & 1.51 & 1.27- \\ \text { during follow-up } & & 1.57 & & 2.74 & & 1.80 \\ \text { Hip or vertebral } & 2.83 & 1.45- & 6.88 & 3.64- & 3.41 & 1.92- \\ \text { fracture during } & & 5.50 & & 13.02 & & 6.06\end{array}$

follow-up corresponding author on reasonable request and subsequent approval by VA Privacy and Information Security Officers.

Samantha L. Solimeo, $\mathrm{PhD}, \mathrm{MPH} \mathrm{H}^{1,2,3,4}$

Brian C. Lund, PharmD, MS

Kimberly D. McCoy, MS ${ }^{1,2}$

Mary Vaughan Sarrazin, $\mathrm{PhD}^{1,2,4}$

Richard Hoffman, $M D, M P H^{2,4,5}$

${ }^{1}$ Comprehensive Access Delivery Research and Evaluation Center, Iowa City VA Health Care System,

Iowa City, IA, USA

${ }^{2}$ Veterans Rural Health Resource Center-Iowa City, VA Office of Rural Health,

Iowa City, IA, USA

${ }^{3}$ Primary Care Analytics Team-Iowa City, VA Office of Patient Care Services,

Iowa City, IA, USA

${ }^{4}$ Department of Internal Medicine, University of Iowa Carver College of Medicine,

Iowa City, IA, USA

${ }^{5}$ Department of Epidemiology, University of Iowa College of Public Health,

Iowa City, IA, USA

Corresponding Author: Samantha L. Solimeo, PhD, MPH; Veterans Rural Health Resource Center-Iowa City, VA Office of Rural Health, Iowa City, IA, USA (e-mail: Samantha.solimeo@va.gov).

Funding SLS received support for this research from VA HSR\&D (Award \#CDA 13-272), the Center for Access \& Delivery Research and Evaluation (CADRE), Department of Veterans Affairs, Iowa City VA Health Care System, Iowa City, IA (Award \#CIN 13-412), the Veterans Rural Health Resource Center Iowa City, VA Office of Rural Health, and the Primary Care Analytics Team-Iowa City, Iowa City VA Health Care System, Iowa City, IA, which is funded by the VA Office of Patient Care Services.

\section{Compliance with Ethical Standards:}

Disclaimer: The views expressed in this article are those of the authors and do not necessarily reflect the position or policy of the Department of Veterans Affairs or the United States government.

\section{REFERENCES}

1. Skolarus TA, Hawley ST. Prostate cancer survivorship care in the Veterans Health Administration. Fed Pract. 2014;31(8): 10-17.

2. Cosman F, de Beur SJ, LeBoff MS, et al. Erratum to: Clinician's guide to prevention and treatment of osteoporosis. Osteoporos Int. 2015;26(7):2045-2047. https://doi.org/10.1007/s00198-015-3037-x

3. Colón-Emeric CS, Pieper CF, Van Houtven CH, et al. Limited osteoporosis screening effectiveness due to low treatment rates in a national sample of older men. Mayo Clin Proc. 2018;93(12):1749-1759.

4. Noonan EM, Farrell TW. Primary care of the prostate cancer survivor. Am Fam Phys. 2016; 93(9):764-770

5. Elixhauser A, Steiner C, Harris D, et al. Comorbidity measures for use with administrative data. Med Care. 1998:36:8-27.

Publisher's Note: Springer Nature remains neutral with regard to jurisdictional claims in published maps and institutional affiliations. course of care

All models were adjusted for year of cohort entry to account for trends in care over time

*Sum of comorbidities identified using the Elixhauser approach ${ }^{4}$ 